\title{
Dialister pneumosintes
}

National Cancer Institute

\section{Source}

National Cancer Institute. Dialister pneumosintes. NCI Thesaurus. Code C122281.

A species of Gram-negative, non-fermenting, anaerobic, rod-shaped bacterium in the family Veillonellaceae. The species was first isolated from nasopharyngeal secretions of patients during the flu epidemic of 1918 to 1921. 